\title{
Can measuring urinary biomarkers improve the management of lupus nephritis?
}

\author{
Anisur Rahman* \\ See related research by Singh et al., http://arthritis-research.com/content/14/4/R164
}

\begin{abstract}
Although renal biopsy is the most accurate way of assessing renal inflammation in patients with lupus nephritis (LN), the technique is invasive and cannot be performed frequently. Currently used blood and urine biomarkers have limited utility in monitoring the activity of nephritis. In a previous issue of Arthritis Research and Therapy, Singh and colleagues showed that measuring urinary levels of vascular cell adhesion molecule 1 could be useful in both diagnosing and monitoring LN. These levels are higher in patients with lupus than controls, are higher in lupus patients who have active renal disease compared with those who do not, and correlate significantly with the histological activity index in renal biopsies of patients with $L N$.
\end{abstract}

Lupus nephritis (LN) is one of the most severe forms of systemic lupus erythematosus (SLE). If not treated adequately, the disease can result in renal failure or death. In its early stages, LN may be almost asymptomatic and picked up only by carrying out routine blood and urine tests. Measurements of urine protein are especially helpful in this regard. In a previous issue of Arthritis Research and Therapy, Singh and colleagues described tests for three different urinary biomarkers in patients with SLE, investigating which is likely to be most helpful for monitoring renal disease activity [1].

Treatment of LN was revolutionized by the introduction of combined corticosteroid/cyclophosphamide regimes and has advanced more recently with the replacement of cyclophosphamide by mycophenolate in many cases [2]. Early diagnosis and treatment has a major impact on clinical outcomes in patients with LN [3], but can also cause treatment-related adverse effects. Being able to

*Correspondence: anisur.rahman@ucl.ac.uk

Centre for Rheumatology Research, 4th Floor, Rayne Institute, 5 University Street, London WC1E 6JF identify exactly when treatment is needed during the course of the disease and when maintenance treatment needs to be increased is therefore important. The most accurate way to assess nephritis in patients with SLE is by carrying out a renal biopsy. The histological type of nephritis can be defined and the degree of inflammation can be quantified using an activity index [4]. A high activity index signifies active yet reversible disease, whereas the chronicity index shows irreversible damage.

Renal biopsy is an invasive procedure, however, which cannot be repeated whenever a flare of renal lupus is suspected. We would therefore like to have biomarkers, measurable in blood or urine, which rise and fall with renal disease activity and are closely associated with the degree of inflammation in the kidney. Blood markers such as anti-double-stranded DNA, anti-nucleosome and anti- $\alpha$-actinin antibodies have been studied. There is some evidence that increases in these markers are associated with renal disease activity [5], as measured by indices such as blood albumin and urine protein, but very little evidence for their association with renal biopsy scores.

Given that the kidney is the main site of inflammation in $\mathrm{LN}$, however, biomarkers in urine may reflect this inflammation more closely than those in the blood. There has been some interest in using urinary neutrophil gelatinase-associated lipocalin as a marker of renal inflammation in SLE. Studies have shown that urinary (but not serum) neutrophil gelatinase-associated lipocalin levels correlate with measures of renal disease activity and that a rise in urinary neutrophil gelatinase-associated lipocalin at one visit predicts flare of nephritis at the next visit $[6,7]$. These studies were cross-sectional and did not look at the association between urinary neutrophil gelatinase-associated lipocalin and renal histology.

The current paper by Singh and colleagues is also a cross-sectional study but includes data on renal histology and compares three different urinary assays; CXCL16, monocyte chemoattractant protein 1 (MCP-1) and vascular cell adhesion molecule 1 (VCAM-1) [1]. Since all three of these molecules can play a role in the recruitment of inflammatory cells to the nephritic kidney, it would be 
entirely reasonable to predict that their levels would rise in the urine when active renal inflammation is at its height. The authors review both clinical and murine evidence supporting this prediction for each of these markers [8]. In this study they report creatinine-normalized urinary CXCL16, MCP-1 and VCAM-1 levels in 73 patients with SLE, 13 healthy volunteers and 22 patients with other forms of glomerulonephritis.

The study results showed some evidence of utility for all three of these urinary markers. All markers were elevated in patients with SLE compared to healthy controls and correlated with the level of proteinuria. However, the comparison with healthy controls is complicated by the fact that $92 \%$ of these controls were Asian whereas none of the patients were. Disease activity in the study was assessed using the Systemic Lupus Erythematosus Disease Activity Index and correlated with MCP-1 and VCAM-1 but not CXCL16. When patients were defined as having active or inactive renal disease, on the basis of the renal components of the Systemic Lupus Erythematosus Disease Activity Index score alone, MCP-1 and VCAM-1 but not CXCL16 distinguished those two groups. However, this result was based on a relatively small number of patients with inactive renal disease $(n=14)$, probably because there was a low threshold for considering a patient to have active renal disease - being positive for any single criterion of hematuria, pyuria, proteinuria or casts was sufficient. This difficulty in defining active LN based on disease activity measures is common to many papers of this type and is a major reason why the availability of renal biopsy data from the date of the urine sample in 24 of these patients is so important. These histological data provide a direct objective measure of nephritis and particularly support the utility of urinary VCAM-1 as a biomarker, since only VCAM-1 was significantly correlated with the activity index and also differentiated class IV LN from the other histological types.

This study thus provides compelling evidence that measuring urinary VCAM-1 could be an important new biomarker in patients with $\mathrm{LN}$, and some evidence supporting urinary CXCL16 and MCP-1. Investigation into whether an index combining the values of all three markers, along the lines of the serum chemokine index reported by Bauer and colleagues [9], would have more potential to distinguish active $\mathrm{LN}$ from inactive $\mathrm{LN}$ would be interesting. As suggested by the authors, however, the most important thing is to study levels of these urinary markers longitudinally in patients with LN to see whether they predict changes in activity of nephritis in individual patients over time.

\section{Abbreviations}

LN, lupus nephritis; MCP-1, monocyte chemoattractant protein 1; SLE, systemic lupus erythematosus; VCAM-1, vascular cell adhesion molecule 1.

\section{Competing interests}

The authors declare that they have no competing interests.

Published: 19 December 2012

\section{References}

1. Singh S, Wu T, Xie C, Vanarsa K, Han J, Mahajan T, Oei HB, Ahn C, Zhou J, Putterman C, Saxena R, Mohan C, Han J: Urine VCAM-1 as a marker of renal pathology activity index in lupus nephritis. Arthritis Res Ther 2012, 14:R164.

2. Contreras G, Pardo V, Leclercq B, Lenz O, Tozman E, O'Nan P, Roth D: Sequential therapies for proliferative lupus nephritis. N Engl J Med 2004, 350:971-980.

3. Fiehn C, Hajjar Y, Mueller K, Waldherr R, Ho AD, Andrassy K: Improved clinical outcome of lupus nephritis during the past decade: importance of early diagnosis and treatment. Ann Rheum Dis 2003, 62:435-439.

4. Austin HA, 3rd, Muenz LR, Joyce KM, Antonovych TT, Balow JE: Diffuse proliferative lupus nephritis: identification of specific pathologic features affecting renal outcome. Kidney Int 1984, 25:689-695.

5. Manson JJ, Ma A, Rogers P, Mason LJ, Berden JH, van der Vlag J, D'Cruz DP, Isenberg DA, Rahman A: Relationship between anti-dsDNA, antinucleosome and anti-alpha-actinin antibodies and markers of renal disease in patients with lupus nephritis: a prospective longitudinal study. Arthritis Res Ther 2009, 11:R154.

6. Pitashny M, Schwartz N, Qing X, Hojaili B, Aranow C, Mackay M, Putterman C: Urinary lipocalin-2 is associated with renal disease activity in human lupus nephritis. Arthritis Rheum 2007, 56:1894-1903.

7. Rubinstein T, Pitashny M, Levine B, Schwartz N, Schwartzman J, Weinstein E, Pego-Reigosa JM, Lu TY, Isenberg D, Rahman A, Putterman C: Urinary neutrophil gelatinase-associated lipocalin as a novel biomarker for disease activity in lupus nephritis. Rheumatology (Oxford) 2010, 49:960-971.

8. Wu T, Xie C, Wang HW, Zhou XJ, Schwartz N, Calixto S, Mackay M, Aranow C, Putterman C, Mohan C: Elevated urinary VCAM-1, P-selectin, soluble TNF receptor-1, and CXC chemokine ligand 16 in multiple murine lupus strains and human lupus nephritis. J Immunol 2007, 179:7166-7175.

9. Bauer JW, Petri M, Batliwalla FM, Koeuth T, Wilson J, Slattery C, Panoskaltsis-Mortari A, Gregersen PK, Behrens TW, Baechler EC: Interferonregulated chemokines as biomarkers of systemic lupus erythematosus disease activity: a validation study. Arthritis Rheum 2009, 60:3098-3107.

doi:10.1186/ar4098

Cite this article as: Rahman A: Can measuring urinary biomarkers improve the management of lupus nephritis? Arthritis Research \& Therapy 2012, 14:127. 